\title{
Determination of Unique Amino Acid Substitutions in Protein Variants by Peptide Mass Mapping with FT-ICR MS
}

\author{
Katsuhiro Tanaka, ${ }^{*}$ Shigeo Takenaka, and Shingo Tsuyama \\ Department of Veterinary Science, Graduate School of Life and Environmental Sciences, Osaka Prefecture \\ University, Osaka, Japan
}

\section{Yoshinao Wada}

Department of Molecular Medicine, Osaka Medical Center and Research Institute for Maternal and Child Health, Osaka, Japan

\begin{abstract}
Peptide mass mapping plays a central role in the structural characterization of protein variants with single amino acid substitutions. Among the 20 standard amino acids found in living organisms, 18, all but Leu and Ile, differ from each other in molecular mass. The mass differences between amino acids range from 0.0364 to $129.0578 \mathrm{Da}$. The mass of the mutated peptide or the difference between normal and mutated peptides uniquely determines the type of substitution in some cases, and even pinpoints the position of the mutation when the involved residue is found only once in the peptide. Among 75 pairs of amino acid residues that are exchangeable via a single nucleotide replacement, 53 show specific change in exact mass, while only 25 in nominal mass. On the other hand, precise measurement, at least to the third decimal place, greatly enhances the capacity of the peptide mass mapping strategy for structural characterization. This notion was verified by an analysis of three $\mathrm{Hb}$ variants using MALDI-FTICR MS. In addition, the baseline resolution of two $1 \mathrm{kDa}$ peptides with a single amino acid difference, Lys or Gln, which have the smallest (0.0364 Da) difference among residues, was achieved by measurement at a mass resolving power of 342,000 . The results indicated that the smallest difference, 0.0040 Da between [ $\Delta 29.9742$ for Glu-Val] and [ $\Delta 29.9782$ for Trp-Arg], among all types of amino acid substitutions derived from a single nucleotide replacement can be discriminated at the present performance level. Therefore, FTICR MS is capable of identifying all 53 types of substitutions, each of which is associated with a unique mass difference, except for the Leu and Ile isomers. (J Am Soc Mass Spectrom 2006, 17, 508-513) (c 2006 American Society for Mass Spectrometry
\end{abstract}

$\mathrm{M}$ any diseases are caused by a single nucleotide substitution in the coding region of the responsible gene. This type of mutation produces mutated proteins with single amino acid substitutions, unless the resulting codon terminates translation or the mutated mRNA or proteins are quite unstable. The mass spectrometric strategy of characterizing the mutated proteins was established a decade ago, mostly utilizing hemoglobin $(\mathrm{Hb})$ variants as a model protein [1-6]. The approach involves two steps: molecular mass measurements of intact proteins [7], and peptide mass mapping of enzymatic digests $[8,9]$. Detection of the molecular mass change caused by an amino acid substitution is an essential part of either analysis.

Published online February 20, 2006

Address reprint requests to Dr. Y. Wada, Department of Molecular Medicine, Osaka Medical Center and Research Institute for Maternal and Child Health, 840 Murodo-cho, Izumi, Osaka 594-1101, Japan. E-mail: waday@mch.pref.osaka.jp

* Also at the Department of Molecular Medicine, Osaka Medical Center and Research Institute for Maternal and Child Health, Osaka, Japan.
Among the 190 pairs formed by the 20 standard amino acids, only an exchange of Leu and Ile results in the same elemental composition, and therefore cannot be characterized on the basis of molecular mass. Other types of exchange accompanying a mass shift from 0.0364 Da for Glu-Lys to 129.04578 Da for Trp-Gly are theoretically amenable to the mass-based strategy. More importantly, according to the point mutation rule, the size of a mass shift uniquely defines the type and position of the substitution in many cases, as discussed in earlier studies $[1,10]$. In addition, another rule of genetics in nature, where the vast majority of amino acid substitutions are caused by single nucleotide substitutions, facilitates the mass-based elucidation, because only $40 \%$ of the 190 different types of amino acid substitutions are derived from a single nucleotide substitution. Although some types of amino acid mutations are indistinguishable from each other because they involve the exact same change in elemental composition, the capability of this strategy depends on the resolution of the measurement. For example, two amino 
Table 1. Mass differences between peptides of wild-type and mutant globins

\begin{tabular}{|c|c|c|c|c|}
\hline & \multirow[b]{2}{*}{ Amino acid } & \multicolumn{2}{|c|}{$m / z$} & \multirow[b]{2}{*}{ Error ppm } \\
\hline & & Observed & Theoretical & \\
\hline Wild & 97His & 1126.5659 & 1126.5645 & 1.25 \\
\hline Moriguchi & 97Tyr & 1152.5703 & 1152.5689 & 1.21 \\
\hline Mass-shift & His-Tyr & -26.0044 & -26.0044 & 0.38 \\
\hline Wild & 92Arg & 1087.6270 & 1087.6264 & 0.64 \\
\hline J Capetown & $92 \mathrm{GIn}$ & 1059.5846 & 1059.5839 & 0.51 \\
\hline Mass-shift & Arg-GIn & 28.0424 & 28.0425 & 4.28 \\
\hline Wild & $22 \mathrm{Glu}$ & 1314.6670 & 1314.6654 & 1.19 \\
\hline G Coushatta & 22Ala & 1256.6616 & 1256.6599 & 1.36 \\
\hline Mass-shift & Glu-Ala & 58.0054 & 58.0055 & 2.14 \\
\hline
\end{tabular}

acid substitution types, Phe-Asp and Tyr-Asp, give the same nominal mass shift of $48 \mathrm{Da}$, but differ from each other by 0.03639 Da being $\Delta 48.00000 \mathrm{Da}$ versus $\Delta 48.03639 \mathrm{Da}$, respectively.

Human $\mathrm{Hb}$ is a tetrameric protein in erythrocytes and is comprised of two globin molecules, $\alpha$ and $\beta$ subunits, each of which bears a prosthetic heme. The subunits of adult $\mathrm{Hb}(\mathrm{Hb} \mathrm{A})$ are composed of 141 amino acid residues $(M r=15,126.4)$ for $\alpha$ and 146 amino acid residues $(M r=15,867.2)$ for $\beta$, respectively. Most of the $\mathrm{Hb}$ variants are caused by an amino acid substitution in one of these subunits and are derived from a single nucleotide change in the gene. In most cases, the exception being highly prevalent mutations such as that causing sickle cell disease, $\mathrm{Hb}$ mutations are heterozygotic and thus both the normal peptide and its mutated counterpart are present in the patient.

Herein, precise measurement of the difference in the masses of normal and mutated peptides was carried out by Fourier transform ion cyclotron resonance (FTICR) MS [11]. The results demonstrate precise measurements to greatly enhance the capability of peptide mass mapping for structural characterization of amino acid substitutions in protein variants.

\section{Materials and Methods}

\section{Materials}

Three hemoglobin variants, $\mathrm{Hb}$ Moriguchi $(\beta 97 \mathrm{His} \rightarrow \mathrm{Tyr})$, $\mathrm{Hb}$ G Coushatta $(\beta 22 \mathrm{Glu} \rightarrow \mathrm{Ala})$, and $\mathrm{Hb}$ J Cape Town $(\alpha 92 \mathrm{Arg} \rightarrow \mathrm{Gln})$, with known structures $[2,4,5]$ were employed in this study. An enzymatic digest of globins was prepared according to methods described previously $[2,10]$. The mutated peptide of $\mathrm{Hb}$ G Coushatta was purified by reversed-phase chromatography for MS/MS.

Synthetic peptides, LQQCPFEDH (1115.4706 Da) and LKQCPFEDH (1115.5070 Da), were purchased from Sigma (St. Louis, MO).

\section{MALDI-FTICR MS}

MS was performed in positive ion mode using a MALDI-FTICR mass spectrometer (IonSpec, Lake For- est, CA) equipped with an actively shielded 7-tesla superconducting magnet (Cryomagnetics, Oak Ridge, TN). An air-cooled Nd:YAG laser system (New Wave, Fremont, CA) was used for ionization and desorption. The sample was mixed with $50 \mathrm{mg} / \mathrm{mL}$ recrystallized 2,5-dihydroxybenzoic acid on a stainless steel target. All mass spectra were acquired in single non-repetitive shot mode followed by a single scan, because multiple shot mode and accumulation of scans impair mass accuracy. Each measurement was repeated 20 times. The protonated molecules for the peptides, except those involved in the mutation, were used for the internal mass calibration.

Fragmentation of the peptides was accomplished by sustained off resonance irradiation and collision-induced dissociation (SORI-CID).

\section{Statistical Analysis}

Confidence intervals (CI) were calculated with Student's $t$-distribution.

\section{Results}

In the peptide mass mapping for detection of mutations, tryptic digestion is most often used and the resulting peptides have a mass of $1000 \mathrm{Da}$ on average. The mutated peptides are $\beta$ T11 (peptide no.11 of tryptic digest of $\beta$-globin chain) for $\mathrm{Hb}$ Moriguchi and $\beta \mathrm{T} 3$ for $\mathrm{Hb} \mathrm{G}$ Coushatta, and the molecular masses for the corresponding wild peptides are 1125.5567 and 1313.6575 , respectively. For $\mathrm{Hb}$ J Cape Town, digestion with lysylendopeptidase was applied, as the substitution of Gln for Arg turns off the tryptic cleavage at the mutated site, and the peptide $\alpha \mathrm{L} 9$ (peptide no. 9 of lysylendopeptidase digest of $\alpha$-globin chain) with an exact molecular mass of 1086.6186 for the wild type is the target in this case. All these mutations are heterozygotic and the variants are present at $\sim 50 \%$, as are their normal counterparts. The FTICR MS of these peptides represented the mass accuracy within a few ppm (Table 1). 

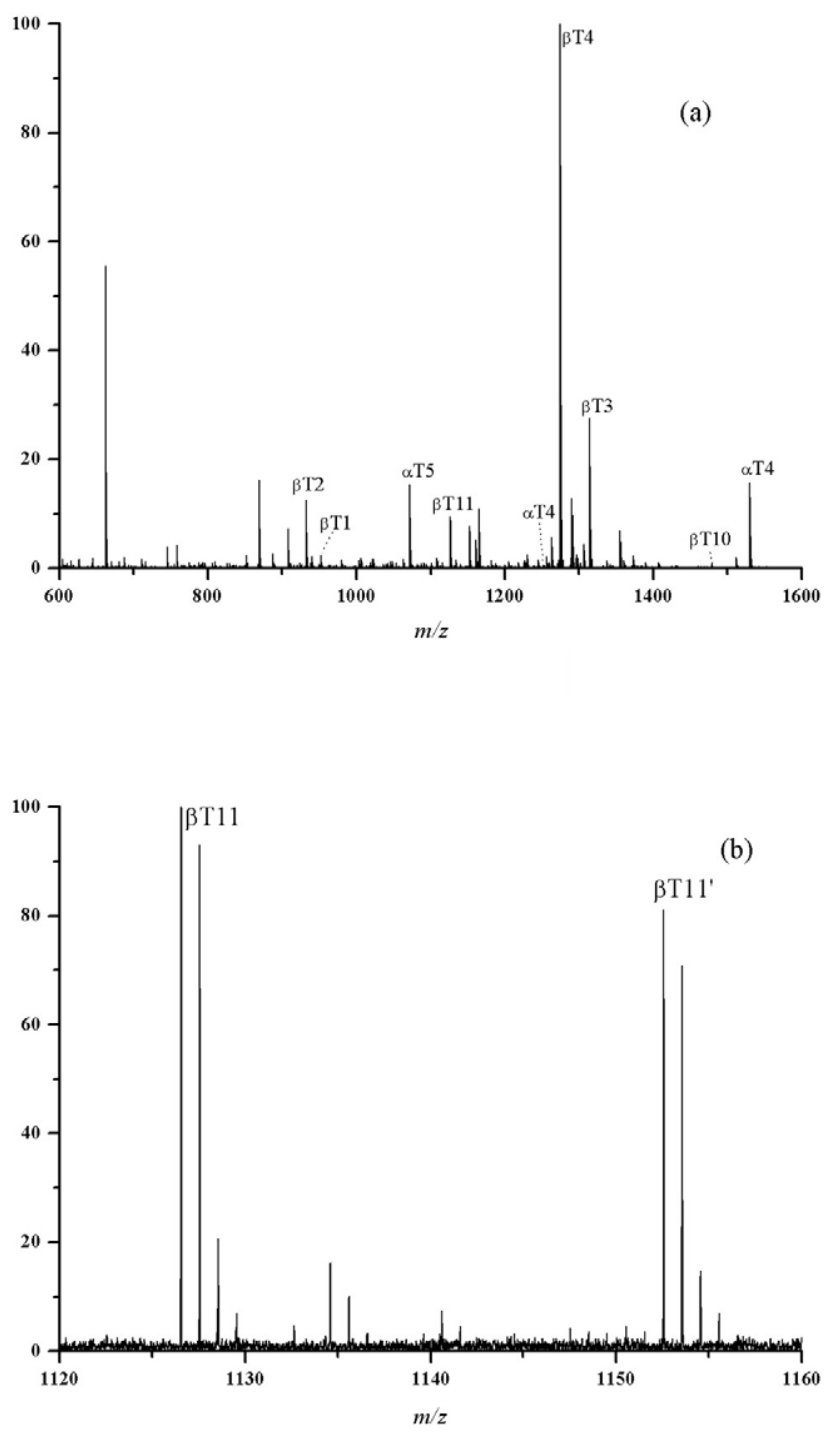

Figure 1. MALDI-FT-ICR mass spectra of tryptic digest of carbamidomethylated $\alpha$ and $\beta$ globins of $\mathrm{Hb}$ Moriguchi. (a) Full spectrum. (b) Depicted spectrum for protonated $\beta$ T11 region. The ions for normal and mutated $\left(\beta \mathrm{T} 11^{\prime}\right)$ peptides are observed at $\mathrm{m} / \mathrm{z}$ 1126.5638 and 1152.5689 , respectively, since the mutation was heterozygotic. Normal $\beta$ T11 sequence is LHVDPENFR.

\section{Hb Moriguchi $(\beta 97 H i s \rightarrow$ Tyr $)$}

The normal sequence of $\beta$ T11 is LHVDPENFR. The MALDI-FTICR mass spectrum of a tryptic digest is presented in Figure 1, and the signals at $m / z 1126.5638$ and 1152.5689 are the ions for wild and mutated $\beta$ T11 peptides, respectively. The difference in nominal mass is $26 \mathrm{Da}$, suggesting five different types of mutations: His $\rightarrow$ Tyr (26.0044), Ala $\rightarrow$ Pro (26.0157), cmCys (carbamidomethylcysteine) $\rightarrow$ Trp (26.0487), Ser $\rightarrow$ Ile (26.0520), and Ser $\rightarrow$ Leu (26.0520) (exact mass in parentheses and unmodified cysteine excluded). In light of the point mutation rule, only His $\rightarrow$ Tyr is ascribed to the molecular mass shift of this peptide, because none of the other residues, Ala, Cys, or Ser, are present in the wild $\beta$ T11 peptide. This assumption can be confirmed statistically by precise measurement of the mass shift as follows.

The mean of 20 mass difference measurements was 26.00439, and the standard deviation was 0.00046 . Therefore, the $99 \%$ confidence interval (CI) is [26.004094, 26.004686], and only Tyr $\rightarrow$ His among the five types of substitutions described above lies within the $99 \%$ CI (Table 2). The position of the mutation was unequivocally demonstrated to be at the single Tyr, the second residue of $\beta$ T11.

\section{Hb J Cape Town $(\alpha 92 \mathrm{Arg} \rightarrow \mathrm{Gln})$}

The normal sequence of $\alpha$ L9 peptide is LRVDPVNFK. In the MALDI-FTICR mass spectrum, the ions at $\mathrm{m} / \mathrm{z}$ 1087.6267 and 1059.5841 are derived from wild and mutated peptides, respectively (Figure 2). The mean of 20 mass difference measurements was 28.04238 Da, and the $99 \%$ CI was [28.041950, 28.042803]. Only the Arg $\rightarrow$ Gln mutation giving the calculated shift of 28.0425 Da lies within the 99\% CI (Table 2), while the nominal mass difference of 28 Da suggested five different types of mutations: Asp $\rightarrow$ Ser (27.9949), Glu $\rightarrow$ Thr (27.9949), Arg $\rightarrow$ Lys (28.0061), Val $\rightarrow$ Ala (28.0313), and Arg $\rightarrow$ Gln. Since one Arg residue is present in the sequence, the substitution is uniquely located at that position.

\section{$H b$ G Coushatta $(\beta 22$ Glu $\rightarrow$ Ala $)$}

The normal sequence of $\beta \mathrm{T} 3$ peptide is VNVDEVGGEALGR. The signals at $m / z 1256.6614$ and 1314.6670 are protonated $\beta$ T3 from mutated and wild globins, respectively (Figure $3 a$ and $b$ ). The difference in nominal mass suggests four different types of mutations: Trp $\rightarrow$ Lys (57.9844), Asp $\rightarrow$ Gly (58.0055), Glu $\rightarrow$ Ala

Table 2. The $99 \%$ Confidence Intervals (CI) for each measured mass-shift and the nearest $(*)$ and the second nearest mass-shifts

\begin{tabular}{|c|c|c|c|}
\hline & $\mathrm{Cl}$ & Theoretical mass shift & Mutation \\
\hline Hb Moriguchi & {$[26.004094,26.004686]$} & $\begin{array}{l}26.0044 \\
26.0157\end{array}$ & $\begin{array}{r}\text { (Tyr-His)* } \\
\text { (Pro-Ala) }\end{array}$ \\
\hline Hb J Cape Town & [28.041776,28.042984] & $\begin{array}{l}28.0313 \\
28.0422\end{array}$ & $\begin{array}{l}\text { (Val-Ala) } \\
\text { (Arg-GIn) }\end{array}$ \\
\hline Hb G Coushatta & {$[58.004628,58.006082]$} & $\begin{array}{l}58.0053 \\
58.0207\end{array}$ & $\begin{array}{c}\text { (Glu-Ala)* } \\
\text { (Trp-GIn) }\end{array}$ \\
\hline
\end{tabular}



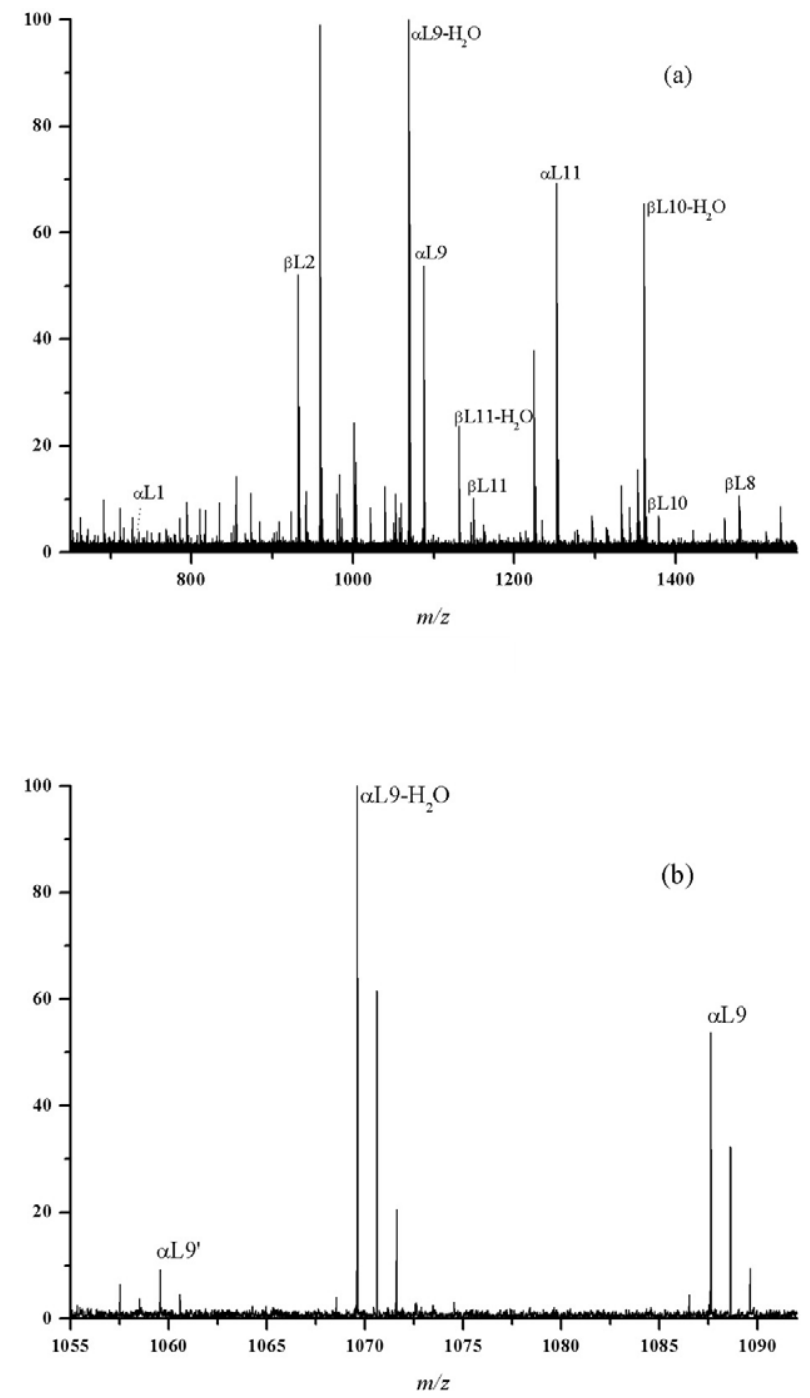

Figure 2. MALDI-FT-ICR mass spectra of the Lys-C digest of carbamidomethylated $\alpha$ and $\beta$ globins of $\mathrm{Hb}$ J Cape Town. (a) Full spectrum. (b) Depicted spectrum for protonated $\alpha \mathrm{L} 9$ region. The ions for normal and mutated $\left(\alpha \mathrm{L} 9^{\prime}\right)$ peptides are observed at $\mathrm{m} / \mathrm{z}$ 1087.6267 and 1059.5841, respectively. Normal $\alpha \mathrm{L} 9$ sequence is LRVDPVNFK.

(58.0055). and $\operatorname{Trp} \rightarrow \mathrm{Gln}$ (58.0207). The measured difference was $58.0054 \pm 0.0014 \mathrm{Da}$, and the $99 \%$ CI was [58.004628, 58.006082]. These results ruled out two types of substitutions, $\operatorname{Trp} \rightarrow$ Lys and $\operatorname{Tr} p \rightarrow$ Gln, leaving two possible mutations, Asp $\rightarrow$ Gly and Glu $\rightarrow$ Ala. Since in total three Asp or Glu candidate residues are present in the sequence of $\beta \mathrm{T} 3$, the type and position of the mutation cannot be uniquely determined solely by the mass shift. SORI-CID was thus used to determine the structure of the mutated peptide in this case. In the MS/MS spectrum shown in Figure $3 c$, the $y_{9}$ ion at $m / z$ 812.4564 contained the Glu $\rightarrow$ Ala mutation at either the fifth or the ninth residue from the N-terminal. Finally, the mutated site was determined to be the former by the MS/MS/MS spectrum, in which the normal mass for the $y_{7}$ ion was generated from the $y_{9}$ ion observed in the MS/MS spectrum (Figure 3d).

\section{Minimal Mass Shift of Gln-Lys in a $1 \mathrm{kDa}$ Peptide}

The smallest mass shift produced by an amino acid substitution is $0.0364 \mathrm{Da}$. This shift is derived from an exchange of Gln for Lys or vice versa. Theoretically, separation of two $1 \mathrm{kDa}$ peptides bearing a mass difference of this size requires a resolution of 30,000, which is readily achievable with a broadband scan. To demonstrate the separation capacity of FTICR MS, two homologous peptides, LQQCPFEDH (1115.4706 Da) and LKQCPFEDH (1115.5070 Da), were synthesized and the mixture was analyzed. The baseline resolution of these peptides was achieved by the MALDI-FTICR mass spectrum at a mass resolving power of 342,000 (Figure 4).

\section{Discussion}

Although structural characterization of protein variants is currently performed by DNA sequence analysis in most cases, MS still plays a central role in characterizing some systemically abundant proteins that can be easily obtained $[12,13]$. A typical example is $\mathrm{Hb}$, a number of mutations of which have been characterized by MS [2, $8,14,15]$. Analysis of the mass of intact proteins is useful for detecting mutations in small proteins such as globins, but the determination of the type and position of such a mutation awaits peptide mass mapping of an enzymatic digest. In analysis of either proteins or peptides, precise measurement of a mass shift derived from the point mutation is essential $[16,17]$, while the Leu and Ile isomers, which can be exchanged with a single nucleotide replacement, are not amenable to this strategy. In the present study, the amino acids with the next smallest difference, Lys and Gln, could be discriminated in a $1 \mathrm{kDa}$ peptide, the average size of tryptic peptides (Figure 4).

In some cases, the type of mutation is uniquely determined by the size of the molecular mass change in peptides, and the site may also be identified when the residue appears only once in the sequence [2]. In this respect, the present study has demonstrated that the precise measurement greatly enhances the capability of peptide mass mapping compared with conventional analysis on a nominal-mass basis. This notion is supported by the following theoretical considerations.

Among 190 different pairs of standard amino acids, 23 have unique differences in nominal mass. For example, His and Glu are the only pair that differs from each other by $8 \mathrm{Da}$, but this type of amino acid substitution requires a double nucleotide replacement in the codon. On the other hand, there are 75 pairs of residues that can be exchanged with single nucleotide substitutions, and only six pairs among them, His-Asp (22 Da), Arg-Cys (53 Da), Arg-Pro (59 Da), Arg-Ser (69 Da), Trp-Cys (83 Da), and Trp-Gly (129 Da), have unique mass differences even on a nominal-mass basis. These six can be readily characterized by straightforward conventional measurements. However, this highlights 

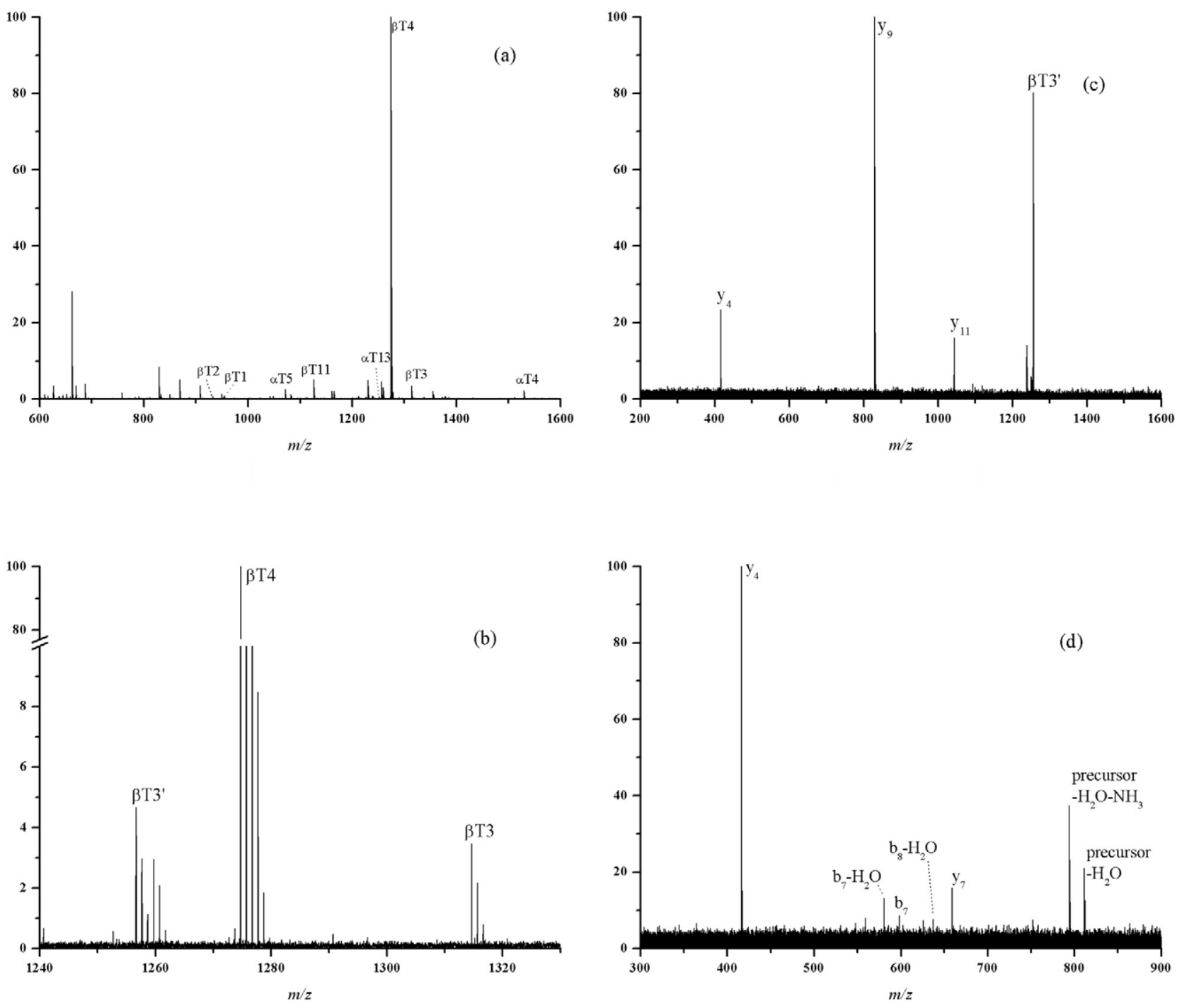

Figure 3. MALDI-FT-ICR mass spectra of the tryptic digest of $\alpha$ and $\beta$ globins of $\mathrm{Hb}$ G Coushatta. (a) Full spectrum. (b) Depicted spectrum for protonated $\beta \mathrm{T} 3$ region. The ions for normal and mutated $\left(\beta \mathrm{T}^{\prime}\right)$ peptides are observed at $\mathrm{m} / \mathrm{z} 1314.6670$ and 1256.6614 , respectively. (c) $\mathrm{MS}^{2}$ product ion spectrum of the purified $\beta \mathrm{T}^{\prime}$. The mutated peptide $\beta \mathrm{T}^{\prime}$ was purified by reversed-phase chromatography and subjected to SORI-CID. Three y-series ions, $\mathrm{y}_{4}, \mathrm{y}_{9}$, and $\mathrm{y}_{11}$ are observed. (d) MS ${ }^{3}$ product ion spectrum of the $\mathrm{y}_{9}$ observed in the MS ${ }^{2}$ spectrum. The normal $\beta \mathrm{T} 3$ sequence is VNVDEVGGEALGR.

that nominal mass measurements do not specify the type of mutation in many cases. For example, there are ten different pairs, Asp-Val, Glu-Leu, Glu-Ile, Cys-Ser, Ser-Ala, Tyr-Phe, Met-Asp, Phe-Met, Leu-Pro, and IlePro, having a difference of $16 \mathrm{Da}$, of which five (AspVal, Cys-Ser, Ser-Ala, Tyr-Phe, and Leu-Pro) can be derived from a single nucleotide substitution. On the other hand, in terms of exact mass, only two, Ser-Ala and Tyr-Phe, in the latter subgroup have the same 15.9949 Da difference. In this way, among a total of 190 pairs, 121 have unique changes in terms of exact mass and, therefore, can be discriminated from each other by precise measurement. When limited to single nucleotide substitutions, 54 (72\%) of 75 types of exchange have specific differences in size (including 0 for Leu-Ile), i.e., in exact mass, while only 25 types (33\%) differ in nominal mass (Figure 5). Among the remaining 21 pairs that cannot be specified on the basis of exact mass, those with a difference of $14.0157 \mathrm{Da}$ for $\mathrm{CH}_{2}$ (Ala-Gly, Thr-Ser, Leu-Val, Ile-Val, and Glu-Asp) constitute the largest group.

Obviously, 54 different sizes of mass shift associated with amino acid substitutions represent the difference in elemental composition of residues. For example, the shift of +26.0044 by the mutation His $\rightarrow$ Tyr of $\mathrm{Hb}$ Moriguchi is calculated from the difference in the compositions of $\mathrm{His}$ and Tyr, $\left[\mathrm{C}_{6} \mathrm{H}_{7} \mathrm{~N}_{3} \mathrm{O}\right]$ versus $\left[\mathrm{C}_{9} \mathrm{H}_{9} \mathrm{NO}_{2}\right]$, namely $\left[\mathrm{C}_{3} \mathrm{H}_{2} \mathrm{O}-\mathrm{N}_{2}\right]$. The smallest difference between amino acids is $0.0364 \mathrm{Da}$, resulting from an exchange of Lys and Gln, and the difference in the 


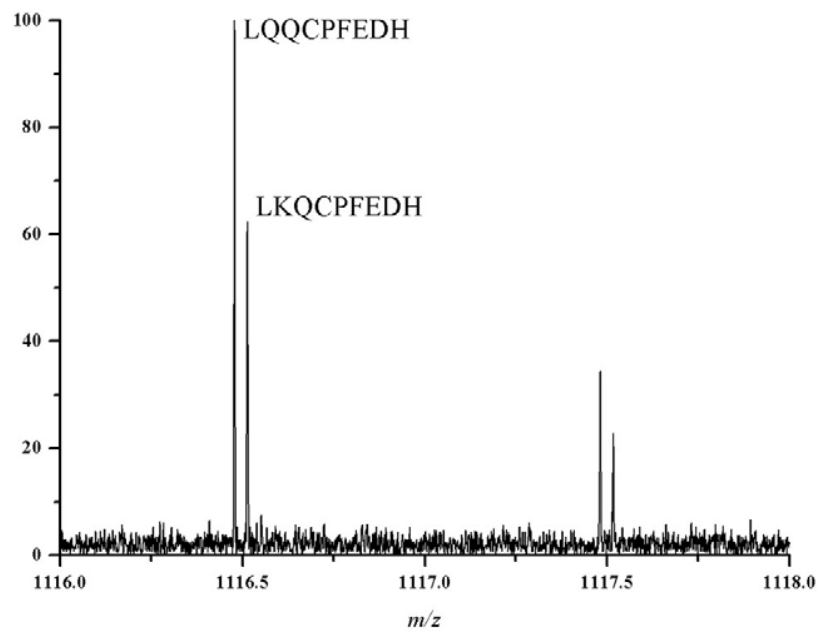

Figure 4. MALDI-FT-ICR mass spectra of a mixture of synthetic peptides with a mass difference of $0.0364 \mathrm{Da}$. The protonated peptides with sequences of LQQCPFEDH and LKQCPFEDH are observed at $\mathrm{m} / \mathrm{z} 1116.4784$ and 1116.5148 , respectively, displaying baseline separation.

elemental composition is $\left[\mathrm{C}_{1} \mathrm{H}_{4}-\mathrm{O}_{1}\right]$. On the other hand, the smallest difference between substitutions (from a single nucleotide replacement) is $0.0040 \mathrm{Da}$, the difference between [ $\Delta 29.9742$ for Glu-Val] and [ $\Delta 29.9782$ for Trp-Arg], and the method described herein can discriminate this small difference as suggested by the accuracy

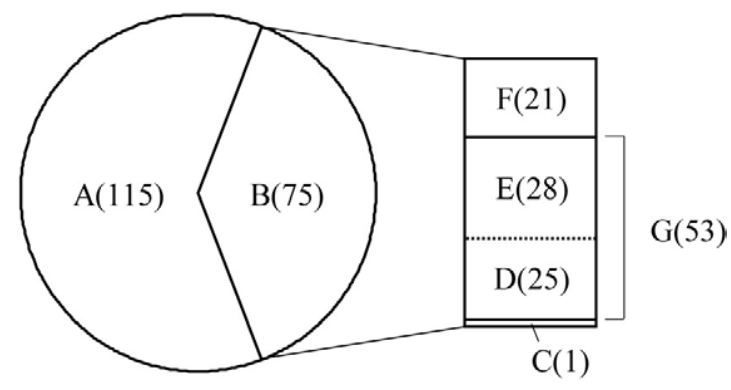

Figure 5. Amino acid replacement pairs with respect to the causative nucleotide substitution and resulting mass shift. There are 190 pairs (Groups A + B) of standard amino acids, and 75 (Group B) can be derived from single nucleotide replacements in the genetic code. Exchange in the Group A pair is extremely rare. In Group B, 54 pairs (Groups C + G) have a specific difference in exact mass between the amino acids involved, and can thus be discriminated from each other, while the others (F) do not. Among the 54 pairs (Groups $C+G), 25$ pairs (Group D) are unique in nominal mass. Therefore, 53 pairs (Group G), the exception being the isomer pair (Group C) of Leu and Ile, are determined by precise measurements. The number of pairs is indicated in parentheses. shown in Table 1. In conclusion, peptide mass mapping by FTICR MS is capable of identifying all 53 types (106 types when $\mathrm{A} \rightarrow \mathrm{B}$ and $\mathrm{B} \rightarrow \mathrm{A}$ are counted separately) of substitutions which are caused by single nucleotide substitutions and have unique mass shifts.

\section{Acknowledgments}

This work was supported by the 21st Century Center of Excellence (COE) Program, Osaka University.

\section{References}

1. Wada, Y.; Hayashi, A.; Fujita, T.; Matsuo, T.; Katakuse, I.; Matsuda, H. Structural Analysis of Human Hemoglobin Variants with Field Desorption Mass Spectrometry. Biochim. Biophys. Acta. 1981, 667, 233-241.

2. Wada, Y.; Matsuo, T.; Sakurai, T. Structure Elucidation of Hemoglobin Variants and Other Proteins by Digit-Printing Method. Mass Spectrom. Rev. 1989, 8, 379-434.

3. Green, B. N.; Oliver, R. W. A.; Falick, A. M.; Shackleton, C. H. L.; Roitmann, E.; Witkowska, H. E. Electrospray MS, LSIMS, and MS/MS for the Rapid Detection and Characterization of Variant Hemoglobins. Burlingame, A. L.; McCloskey, J. A., Eds.; In Biological Mass Spectrometry; Elsevier: Amsterdam, 1990; pp 129-146.

4. Wada, Y.; Matsuo, T.; Papayannopoulos, I. A.; Costello, C. E.; Biemann, K. Fast Atom Bombardment and Tandem Mass Spectrometry for the Characterization of Hemoglobin Variants Including a New Variant. Int. J. Mass Spectrom. Ion Processes 1992, 122, 219-229.

5. Wada, Y.; Matsuo, T. Structure Determination of Aberrant Proteins. In Biological Mass Spectrometry; John Wiley and Sons: Chichester, UK, 1994; pp 369-399.

6. Wada, Y. Advanced Analytical Methods for Hemoglobin Variants. J. Chromatogr. B Anal. Technol. Biomed. Life Sci. 2002, 781, 291-301.

7. Yang, L.; Lee, C. S.; Hofstadler, S. A.; Pasa-Tolic, L.; Smith, R. D. Capillary Isoelectric Focusing-Electrospray Ionization Fourier Transform Ion Cyclotron Resonance Mass Spectrometry for Protein Characterization. Anal. Chem. 1998, 70, 3235-3241.

8. Shimizu, A.; Nakanishi, T.; Nishikawa, M.; Miyazaki, A. Detection and Identification of Protein Variants and Adducts in Blood and Tissues: An Application of Soft Ionization Mass Spectrometry to Clinical Diagnosis. J. Chromatogr. B Anal. Technol. Biomed. Life Sci. 2002, 776, 15-30.

9. Bruce, J. E.; Anderson, G. A.; Wen, J.; Harkewicz, R.; Smith, R. D. High-Mass-Measurement Accuracy and 100\% Sequence Coverage of Enzymatically Digested Bovine Serum Albumin from an ESI-FTICR Mass Spectrum. Anal. Chem. 1999, 71, 2595-2599.

10. Wada, Y. Detection and Characterization of Protein Mutations by Mass Spectrometry. The Protein Protocols Handbook, 2nd ed.; Humana Press Inc: Totowa, NJ, 2002; pp 681-692.

11. Marshall, A. G.; Hendrickson, C. L.; Jackson, G. S. Fourier Transform Ion Cyclotron Resonance Mass Spectrometry: A Primer. Mass Spectrom. Rev. 1998, 17, 1-35

12. Lay, M. J.; Wittwer, C. T. Real-Time Fluorescence Genotyping of Factor V Leiden During Rapid-Cycle PCR. Clin. Chem. 1997, 43, 2262-2267.

13. Bergen, H. R., III; Zeldenrust, S. R.; Butz, M. L.; Snow, D. S.; Dyck, P. J.; Dyck, P. J.; Klein, C. J.; O’Brien, J. F.; Thibodeau, S. N.; Muddiman, D. C. Identification of Transthyretin Variants by Sequential Proteomic and Genomic Analysis. Clin. Chem. 2004, 50, 1544-1552.

14. Wajcman, H.; Prehu, C.; Bardakdjian-Michau, J.; Prome, D.; Riou, J.; Godart, C.; Mathis, M.; Hurtrel, D.; Galacteros, F. Abnormal Hemoglobins: Laboratory Methods. Hemoglobin 2001, 25, 169-181.

15. Wild, B. J.; Bain, B. J. Detection and Quantitation of Normal and Variant Hemoglobins: An Analytical Review. Ann. Clin. Biochem. 2004, 41, 355-369.

16. Lewis, J. K.; Bendahmane, M.; Smith, T. J.; Beachy, R. N.; Siuzdak, G. Identification of Viral Mutants by Mass Spectrometry. Proc. Natl. Acad. Sci. U.S.A. 1998, 95, 8596-8601.

17. Nepomuceno, A. I.; Mason, C. J.; Muddiman, D. C.; Bergen, H. R., III; Zeldenrust, S. R. Detection of Genetic Variants of Transthyretin by Liquid Chromatography-Dual Electrospray Ionization Fourier-Transform Ion-Cyclotron-Resonance Mass Spectrometry. Clin. Chem. 2004, 50, 1535-1543. 